\title{
A pilot peer to peer structured group education program in patients with diabetes mellitus type 1. The Greek experience.
}

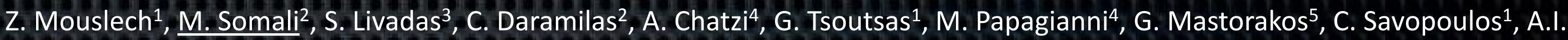
Hatzitolios ${ }^{1}$.

\begin{abstract}
${ }^{1} 1$ st Medical Propedeutic Dept. of Internal Medicine-AHEPA University Hospital-Aristotle University of Thessaloniki-, Endocrinology, Thessaloniki, Greece. 2Euromedica General Clinic, Endocrinology-Metabolism and Diabetes, Thessaloniki, Greece.

${ }^{3}$ Metropolitan Hospital, Endocrine Unit, Athens, Greece.

43rd Pediatric Department-Aristotle University-Hippokration Hospital, Endocrine Unit, Thessaloniki 54642-Greece., Greece.

${ }^{5}$ Aretaeio Hospital-School of Medicine- National and Kapodistrian University Athens, Endocrinology-Metabolism and Diabetes, Athens, Greece.
\end{abstract}

Background: Diabetes mellitus type 1 constitutes a real challenge in everyday practice for both physicians and patients. The complexity of the disease and its unpredictable nature require structured education and training programs that promote active patient involvement and self-care behaviors in order to achieve adequate glycemic control, prevent diabetic complications and improve the quality of life of patients. Along with the necessary knowledge and skills to self-monitor and self-manage the disease and its associated metabolic conditions, peer to peer educational programs have been considered highly successful in motivating and empowering patients with type 1 diabetes mellitus.
Method: The education group (EG) was comprised of 62 patients ( 45 males) with type $1 \mathrm{DM}$, mean age $36 \pm 4.2$ years and BMI: $24.2 \pm 3.1 \mathrm{~kg} / \mathrm{m} 2$. An age- and BMl-matched control group (CG, $n=25$, mean age $41 \pm 6.4$ years, BMl: $25.7 \pm 4.2 \mathrm{~kg} / \mathrm{m} 2$ ) was composed of patients referred but not enrolled in the project. Two hour sessions took place fortnightly in groups of 6-8 T1DM patients the physician in charge (endocrinologist or internist with experience in DM) and a diabetes educator. The educator was a health sciences professional suffering from T1DM. The diabetes regimen remained unchanged throughout the program, with the exception of slight adjustments in insulin dosing (up to $20 \%$ of the total insulin dose). All participants completed the 1-year educational course.

Aim: To evaluate the effect of a structured 12-month peer to peer structured education program that aimed to motivtae patients to acquire better knowledge of the nature of diabetes, follow a healthy decreased $(8.5 \pm 2.1 \% v s .7 .08 \pm 0.79 \%, p<0.0001)$ as was also the incidence Mediterranean diet and exercise regularly as well as to adjust carbohydrate intake and insulin dose according to their needs, self monitor their glucose levels and manage hypo and hyperglycemia in their every-day life.

the above parameters remained stable.

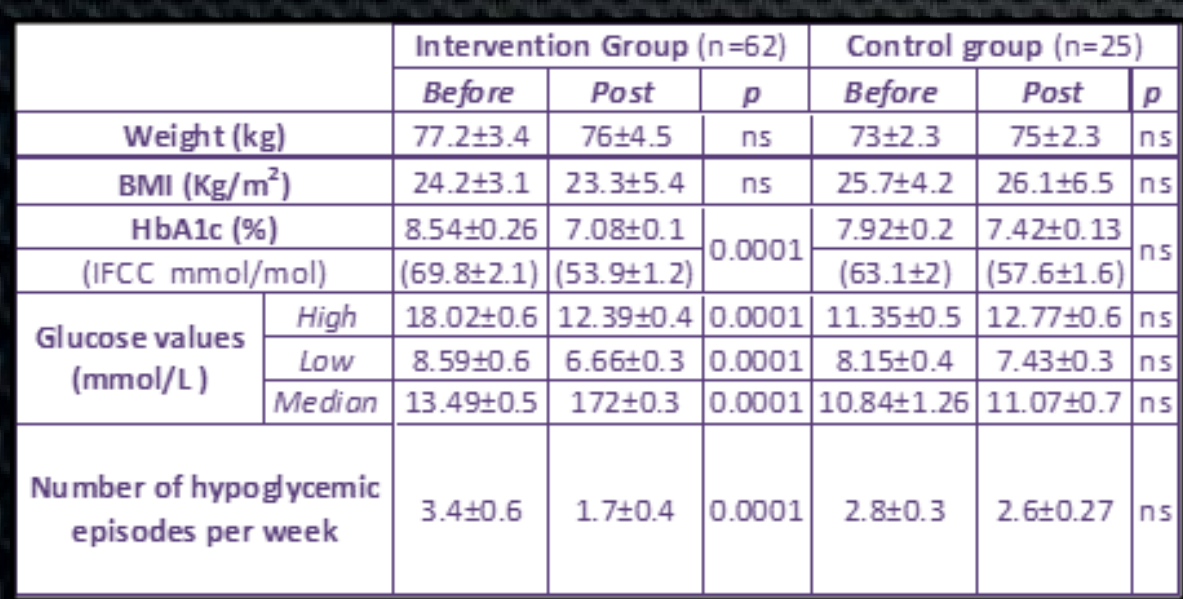

Results of comparison between groups.

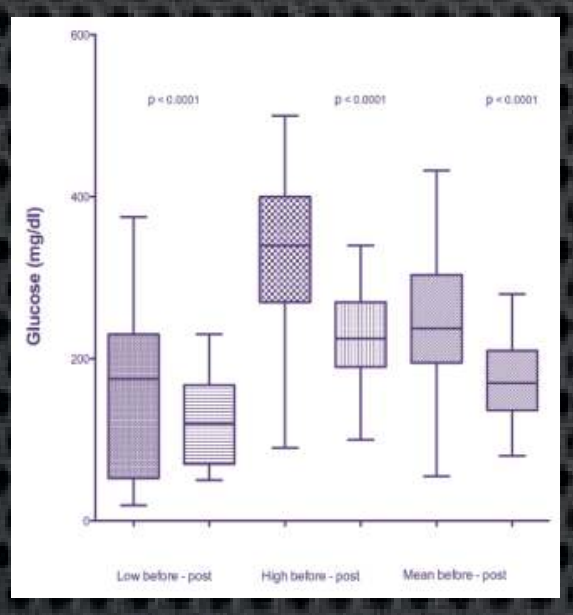

Figure 1: Comparison of high, low and median glucose levels before and after intervention

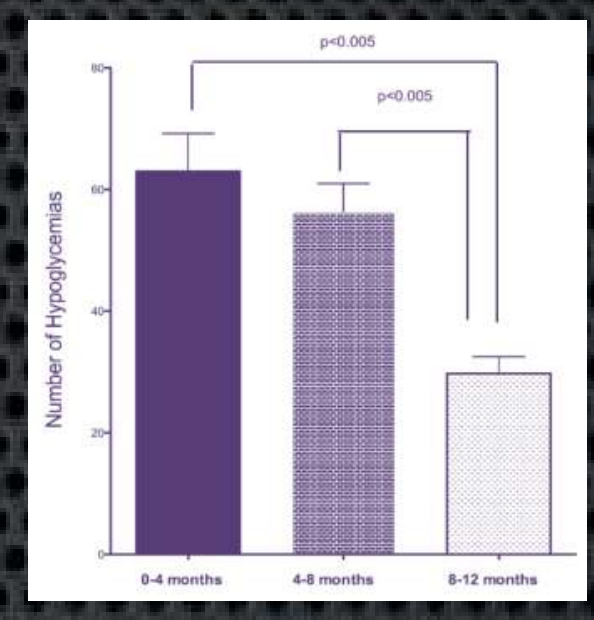

Figure 2: Incidence of hypoglycemic episodes through time in the intervention group

Discussion: These results strongly support the need for long-lasting peer to peer structured education group courses in adult diabetic patients keen to change their habits and acquire better and deeper knowledge about their condition in order to achieve a higher level of awareness and become better equipped with skills to self-manage their diabetes. 